\title{
El discurso de la Unión Europea en materia de políticas de empleo y exclusión social. Análisis sociológico de la Estrategia Europea de Empleo
}

\author{
Antonio Martínez López \\ Departamento de Sociología de la Universidad de Granada. \\ Facultad de Ciencias Políticas y Sociología \\ antmart@ugr.es
}

Recibido: 30-03-2008

Aceptado: 01-07-2008

\section{Resumen}

Especialmente desde mediados de los años noventa, asistimos en Europa a la puesta en marcha y a la consolidación de lo que parece ser una nueva filosofía y su consecuente praxis a la hora de enfrentar las problemáticas que plantea la cuestión social de nuestro tiempo. Las altas tasas de paro de principios de los ochenta, que, mantenidas en el tiempo, dejaron de ser analizadas como coyunturales o friccionales, para aceptar su carácter estructural, han llevado a tomas de posición político-administrativas para resolver las cuestiones del paro y de la exclusión social. La Estrategia Europea de Empleo, implantada y difundida por la Comisión Europea entre los estados miembros, parece ser el instrumento central de este nuevo enfoque. Sustentando el grueso de su filosofía y de sus formas de intervención en la aplicación de políticas activas de empleo, que nacieron en el marco de la economía laboral sueca para resolver problemas de paro friccional, las políticas activas pasan al centro de la acción social contemporánea, interpelando, tal y como planteamos aquí, las formas del Estado de bienestar keynesiano clásico y apuntando hacia la reconstrucción de nuevas representaciones sobre las visiones legítimas de la intervención social en materia de paro y exclusión.

Palabras clave: sociología; sociología del trabajo; Estado de bienestar; políticas sociales; Unión Europea; trabajo social; exclusión social.

Abstract. The discourse of the European Union on employment policies and social exclusion. A social analysis of the European Employment Strategy

From middle of the nineties, in Europe we can see the consolidation of what seems to be a new philosophy and its consistent practice, in relation to facing the problematics of the social question of our time. The high unemployment rates of the beginning of the eighties which later maintained high, weren't analyzed anymore as a conjuntural problem, but being seen as of a structural character. For this reason, politician-administrative officer have led to the point to solve the questions of unemployment and social exclusion. The European Strategy of the Employment, well-established and spread by the European Commission between the member states, seems to be the central instrument of this new approach. Sustaining the force of its philosophy and of its forms of intervention in the application of active policies of employment, that were created in the context of the Swedish labour economy to solve problems of frictional unemployment, the active policies are now at the center of the contemporary social action. They sup- 
pose a change in the forms of intervention of the Keynesian Welfare State, and focus on the construction of new representations of the perspectives ofunemployment and social exclusion.

Key words: sociology, sociology of labour, Welfare State, social policies, European Union, social work, social exclusion.

\section{Sumario}

1. Introducción, objetivos y metodología

2. La consolidación de un discurso europeo o un lenguaje común en materia de empleo y políticas sociales

3. La visión europea de los estados de bienestar activos
4. Algunos balances sobre la Estrategia Europea de Empleo

5. Conclusiones

Referencias bibliográficas

\section{Introducción, objetivos y metodología}

La Estrategia Europea de Empleo está teniendo no pocos impactos, tanto en el discurso europeo sobre lo que es el paro y la forma de combatirlo, como en la "socialización» en este discurso de los distintos actores nacionales que participan en el diseño y el desarrollo de estas políticas (Barbier, 2004).

Dice también el autor francés mencionado:

Una de las influencias más importantes de la Estrategia Europea de Empleo concierne al discurso político. Ha favorecido una cierta convergencia discursiva en torno a un referente político, económico y social, el cual encuentra su base en el paradigma económico dominante, que ha roto básicamente con el ideario keynesiano. (Barbier, 2004: 5)

El autor parte, en este trabajo, de un axioma con el que me solidarizo y creo que da sentido e interés a este trabajo, y es el de considerar que este «lenguaje común» tiene efectos socializadores sobre las élites político-administrativas encargadas de defenderlo a nivel nacional, regional y local.

Así, Barbier (2004: 10) dice que puede definirse la Estrategia Europea de Empleo como un conjunto de actividades fundadas sobre un contenido reglamentario (las disposiciones de los tratados que son obligatorias para todos los estados miembros) y un cuadro cognitivo (una serie de objetivos organizados en cuatro pilares clave, que aquí detallaremos después). Es en relación con ese cuadro cognitivo que gravita este artículo.

El objetivo general que se persigue en el presente escrito es el de reconstruir el discurso europeo que, en materia de empleo y exclusión social, está generando la Comisión Europea y que se produce a raíz de los acuerdos y los tratados a los que se llega en las distintas cumbres europeas que se vienen sos- 
teniendo. Se trata, por tanto, de desvelar un conjunto de representaciones, portadoras de una determinada visión del mundo, que constituyen ese lenguaje común socializador del que habla el autor que he tomado como soporte introductorio.

En definitiva, el presente artículo produce una serie de desarrollos que permiten responder a las siguientes subpreguntas, cuya formulación hay que situar en el origen del propio texto, a saber: ¿cuál es el paradigma económico y social que puede hallarse tras los textos de la Comisión Europea?, ¿qué visión del mercado de trabajo transmiten estas políticas?, ¿existe algún tipo de conexión entre la política económica que se persigue (las grandes líneas directrices de la economía europea) y estas políticas de empleo que se definen y se defienden en la Estrategia Europea de Empleo?, ¿qué tipo de representaciones sobre el paro, sobre los parados y sobre la forma de actuar en torno a este fenómeno se difunden desde la Unión Europea? y, resumiendo prácticamente a todas las anteriores: ¿cuáles serían los paradigmas del modelo de Estado de bienestar a que apuntan estas políticas de empleo?

Por lo que respecta a la metodología que se ha empleado para realizar este trabajo, ha consistido en una selección de los libros escritos por los especialistas más reconocidos a nivel europeo en esta materia, de los documentos forjados por la propia Comisión, sin olvidar los informes de evaluación emanados de requerimientos de la Comisión Europea a distintos intelectuales, que han trabajado en el intento de evaluar estas políticas de empleo y exclusión social, como es el caso del propio Jean Claude Barbier, Serge Paugam, Anne Diamantoupoulou ${ }^{1}$ y otros autores, entre ellos, algunos españoles.

Para el trabajo, se emplean, por supuesto, textos y referencias a textos emanados de la propia Comisión Europea que están en relación con el desarrollo del enfoque sobre políticas sociales y de empleo de la Comisión, y aquí tenemos que señalar que no se han utilizado todos y cada uno de los textos producidos en la instancia mencionada, simplemente porque habría sido imposible, así como altamente innecesario. En este sentido, se ha acotado metodológicamente el periodo en el que puede considerarse que se produce tanto el origen como la consolidación del discurso europeo que se analiza, que iría desde el libro blanco de Delors (1993), hasta la Cumbre de Estocolmo del 2000, esencialmente. Eso sí, de cara a ver cuál ha sido la evolución de este discurso una vez en fase de fuerte consolidación, fase en la que podríamos considerar que nos encontraríamos incluso en estos momentos, se utilizan informes de evaluación de la Unión Europea posteriores.

1. Jean Claude Barbier es economista y sociólogo, antiguo alumno de la Escuela des Hautes Études Commerciales, es profesor en París VII y director de investigaciones en el Centre d'Études sur l'Emploi, así como uno de los especialistas europeos más destacados en el dominio de las políticas activas de empleo. Anne Diamantoupoulou era comisaria de Empleo y Asuntos Sociales en la época en que se emitió el informe que cito. Serge Paugam es maître de conferences y director de investigaciones en el CNRS. 


\section{La consolidación de un discurso europeo o un lenguaje común en materia de empleo y políticas sociales. Centramiento en las políticas activas de empleo}

Una de las hipótesis centrales que se pueden mantener en este documento es que, desde la Unión Europea, se está construyendo y difundiendo un discurso sobre el paro, el empleo y sobre la Europa social que contiene, de manera explícita e implícita, una teoría acerca de cuáles son las principales transformaciones sociales que se están produciendo y las problemáticas sociales más relevantes, y también propone una serie de medidas sociales y políticas para enfrentarse a ellas. Éstas implican fundamentalmente la apuesta por las políticas activas de empleo frente a las consideradas caducas políticas pasivas, como forma principal de tratamiento de la cuestión social de nuestro tiempo. Este discurso supone, visto desde esta perspectiva, tanto una redefinición de conceptos como el Estado de bienestar o la cohesión social, como la aplicación de nuevas metodologías y políticas para la acción social y, en concreto, para abordar la cuestión de la integración sociolaboral de los ciudadanos. En definitiva, podría sostenerse que se está construyendo, a través de la Estrategia Europea de Empleo, un nuevo enfoque en el tratamiento de lo que Robert Castel (1997) llama «la cuestión social».

Fue en el Consejo Europeo de Amsterdam de 1997 donde se consolidó de manera inequívoca la visión que, sobre la política de empleo, ya se había perfilado en el libro blanco sobre crecimiento, empleo y competitividad ${ }^{2}$. En él se reafirmó y se aprobó el título sobre el empleo (título VIII, que pasó de ser un capítulo de un título, en el Tratado de Maastricht, a un capítulo con naturaleza propia en el Tratado de Amsterdam), de forma que se reconocía el empleo como un tema de interés común, tal y como ya se pedía en Maastricht, y se ahondaba en su importancia. Así, el desarrollo de ese nuevo título sobre el empleo se ha ido plasmando en las sucesivas cumbres de Luxemburgo, Cardiff, Colonia, Lisboa, Niza y otras, donde se van consolidando los llamados «procesos por el empleo» de la Estrategia Europea de Empleo (Consejo Económico y Social, 2001:5).

A continuación, abordamos cómo se configura el discurso de la UE en torno a dos ejes que hemos considerado claves para estructurarlo, porque suponen las dos grandes esferas que actúan de mediadoras entre las situaciones de inclusión y de exclusión de los individuos: el mercado de trabajo (discurso que se articula en torno al concepto de empleo y de activación en el empleo, en definitiva: empleabilidadad), y el Estado de bienestar (discurso que se articula en torno al concepto de activación ciudadana).

2. En este sentido, el hito que supone el libro blanco, como punto de arranque de una visión europea que hace del empleo un tema de interés central y lo coloca en el centro del enfoque común europeo a venir, tanto desde el punto de vista ideológico como analítico y político, tal y como se reconoce expresamente en el documento: Comisión Europeénne (2006), Principales étapes de la SEE, Bruselas, Travail et Affaires Sociales. 


\subsection{La visión europea del mercado laboral}

Una de las conclusiones más importantes que pueden extraerse del análisis de los distintos documentos europeos mencionados, es que la filosofía que anima a la Estrategia Europea de Empleo es la de entender la integración social como integración laboral y, por lo tanto, consiste en la construcción de toda una serie de conceptualizaciones y de políticas de inserción que giran en torno a reconstruir y remodelar los mercados de trabajo. Por lo tanto, puede hablarse de la preconización, por parte de la Unión Europea, de un enfoque de la inserción a través de lo económico. De hecho, así lo entiende también Taylor-Gooby (2004: 84), quien, junto con Anne Daguerre, sostiene, en el libro citado, que la Estrategia Europea de Empleo ha de entenderse como un énfasis en las políticas de integración en el mercado de trabajo basadas en la mejora de la formación y las oportunidades de ocupación, todo ello enlazado con ciertas medidas para hacer que el trabajo asalariado resulte más atractivo a las fracciones de la clase obrera que disfrutan de ayudas sociales. A continuación, vemos cómo se va desarrollando esta Estrategia Europea de Empleo.

Meses más tarde de la Cumbre de Amsterdam, se celebra la Cumbre de Luxemburgo de 1997, donde los jefes de estado y de gobierno europeos adoptaron el primer grupo de directrices para el empleo, con el objetivo de desarrollar una política del mercado laboral más activa que en el pasado, tal y como ellos declaraban (Consejo Económico y Social, 2001: 6). En términos de desempleo, se trataba de adoptar estrategias que no se centraran únicamente en el mantenimiento de los ingresos de los parados, lo cual se consideraba una política pasiva en materia de empleo, sino que la Estrategia Europea de Empleo debía de plasmarse en medidas preventivas que hicieran hincapié en ayudar a las personas desempleadas.

La Estrategia Europea de Empleo, que se consolida en la Cumbre de Luxemburgo, se establece en aquel encuentro sobre cuatro pilares:

1) Mejorar la capacidad de inserción profesional.

2) Fomentar el espíritu de empresa.

3) Fomentar la capacidad de adaptación de los trabajadores a las empresas (flexibilizar los mercados, básicamente).

4) Fomentar las políticas de igualdad de oportunidades en el mercado de trabajo.

Los pilares mencionados se encuentran en interconexión a la hora de estructurar el discurso de la Unión Europea y de formular propuestas de acción social, y en su desarrollo se construye un concepto nuclear de la Estrategia Europea de Empleo y de las actuales políticas sociales y de empleo en Europa, y sobre el que se diría que las ciencias sociales no han reparado aún de manera suficiente a fin de comprenderlo y analizarlo. Me refiero al concepto de empleabilidad.

En el Consejo Europeo Extraordinario de Lisboa, de marzo del 2000, que llevaba por título Hacia la Europa de la innovación y el conocimiento, se constituye la llamada Estrategia de Lisboa, que viene a reforzar los pilares de la Estrategia 
Europea de Empleo que se consolidan en Amsterdam y Luxemburgo, pero, además, supone un hito en este proceso, por cuanto comienza a conectarse la estrategia económica y de competitividad con el empleo y la cohesión social, como detallaremos más profundamente en el apartado donde se analiza la evolución del modelo social europeo.

Verificamos aquí una de las constantes en la evolución de la Estrategia Europea de Empleo, que es la de apostar por un modelo de política económica y por un modelo de política de empleo, a pesar de reconocer que el crecimiento económico producido no consigue traducirse correlativamente en términos de colocación en el mercado laboral. En este sentido, ya hemos visto que este fue uno de los reconocimientos explícitos de los que parte el libro blanco de Delors, y que hemos considerado el arranque genuino de las propias políticas activas de empleo como base de una estrategia europea en esta materia. También en Lisboa se reconoce, en primer lugar, el excelente momento que vive Europa en el año 2000 en cuanto a crecimiento económico, a la vez que se señalan los que se consideran los retos con los que tiene que enfrentarse Europa, como son, fundamentalmente, el aumento de la competitividad económica internacional, debido a la universalización o a la globalización de la economía, y la necesidad de mejorar los logros conseguidos en términos de tecnologías de la información y de sociedad del conocimiento para no perder el tren de la competitividad.

A fin de corroborar los argumentos expuestos, a continuación, exponemos las debilidades de la creación de empleo y los peligros que el Consejo de Lisboa señala. Se dice que las debilidades del mercado laboral europeo continúan presentando dificultades en:

- Insuficiencia de la creación de trabajo en el sector de servicios, cuando éste es, con mucho, el más importante desde el punto de vista del empleo.

- Importantes desequilibrios regionales, en particular desde la ampliación en 2004.

- Tasa de desempleo de larga duración elevada.

- Falta de adecuación entre la oferta y la demanda de mano de obra, circunstancia bastante frecuente en períodos de recuperación económica.

- Insuficiente participación de las mujeres en el mercado laboral.

- La evolución demográfica europea y, principalmente, el envejecimiento de la población.

O sea, que, como avance de lo que será una tónica general, se da, por una parte, un espaldarazo a la Estrategia Europea de Empleo tal y como había sido formulada en Amsterdam y Luxemburgo, y, por otra, se reconocen insuficiencias, deficiencias y rémoras en el paso de los beneficios del crecimiento económico al ámbito de la mejora en el mercado de trabajo, pero rémoras y límites que sirven para reafirmar y consolidar la estrategia iniciada, y no para variar su rumbo. Vemos como se reconoce que no se crea suficiente empleo, especialmente en el sector servicios, que es además el sector más dinámico en la economía moderna europea, de ahí que esto sirva para proponer medidas 
de fomento de la formación y educación permanente (en definitiva, medidas vinculadas a las políticas activas de empleo).

Luego se propone que Europa movilice todos sus recursos para crear una economía integradora socialmente, y moderna, basada en los principios de la sociedad del conocimiento. La hipótesis de la que se parte es que la aplicación de los principios tecnológicos de una sociedad del conocimiento al mercado laboral, y especialmente al sector servicios, donde se detecta el mayor retraso en creación de empleo, serviría de acicate para liberar nuevos yacimientos de empleo y abundante cantidad de oportunidades laborales ${ }^{3}$.

En definitiva, en Lisboa se trataba de consolidar la Estrategia Europea de Empleo enfrentándose a una serie de retos relativos a la globalización económica, que eran, tal y como se enuncia también en Consejo Económico y Social (2001: 14), los siguientes:

1. Adecuación de las políticas macroeconómicas y consecución del pleno empleo en el año 2010, lo que supone una tasa media de actividad del 70 por 100 de la población en edad de trabajar y una tasa de empleo, exclusivamente friccional, de un 3-4 por 100.

2. Consolidación de un mercado europeo más competitivo que el de los Estados Unidos, donde se dé cobertura al desarrollo del capital humano y de la nueva economía de la innovación y del conocimiento.

3. Modernización de los sistemas de protección social y promoción de mecanismos dinámicos de protección de empleo.

En la Cumbre de Niza, de diciembre del 2000, se habla de la necesidad de aplicar esta estrategia de empleo al ámbito local. Vuelve a insistirse en la necesidad de invertir en formación permanente, lo que, sin duda, también está en relación con ese déficit detectado en las cualificaciones y que es visto como un obstáculo para la creación de empleo. El Consejo Europeo de Estocolmo, celebrado en marzo de 2001, se centró fundamentalmente en cómo modernizar el modelo europeo y alcanzar el objetivo estratégico de la Unión Europea para el próximo decenio fijado en Lisboa, que es el de convertirse en la economía basada en el conocimiento más competitiva y dinámica del mundo.

Es interesante destacar que, en Estocolmo, se refuerza en el discurso un elemento que ya se había apuntado en Lisboa, y es el de la conexión de lo económico con lo social, del empleo con la exclusión e integración, de las políticas económicas con las políticas sociales. De hecho, se produjo un acuerdo total en que la reforma económica y las políticas sociales y de empleo se refuerzan mutuamente (Consejo Económico y Social, 2001).

En este consejo se enfatiza el compromiso de lograr el pleno empleo, objetivo que se vincula a la necesidad de mantener y modernizar el Estado de bienestar. Como veremos, es esta conexión entre las políticas activas de empleo

3. Comunicación de la Comisión (2000), Políticas comunitarias de apoyo al empleo, Bruselas, COM 78 final. 
y las políticas sociales, y mayormente el llamamiento a la reformas de los estados de bienestar, en el sentido de hacer girar la intervención social sobre la base del acceso al trabajo en la economía de mercado, lo que constituye el armazón teórico central del nuevo proyecto social europeo, tal y como se defiende en el presente artículo. Esta hipótesis que sostenemos aquí sobre la naturaleza de las políticas sociales y de empleo de la Unión Europea y su subordinación a lo económico en la actualidad, se vería también reforzada desde el ámbito de la teoría social por los argumentos de Bob Jessop, citado en Gough (2000: 181), donde defiende que si el fordismo se caracterizaba por el dominio del concepto de Estado de bienestar keynesiano, y las políticas redistributivas, en el ámbito de los mercados laborales, el postfordismo actual supondría la emergencia de un "workfare schumpeteriano", que subordina la política social a las necesidades de un mercado laboral flexible.

\subsection{Los postulados sobre la empleabilidad en el discurso de la Unión Europea}

En primera instancia, hay que destacar el lugar preeminente que se le concede al empleo como eje de integración social. Podemos decir que se hace de la inserción por lo económico, a través del empleo, el pilar de la inserción social ${ }^{4}$. Este enfoque de la empleabilidad implica la asunción de que el mercado de trabajo, a pesar de las limitaciones que se le reconocen ${ }^{5}$, sigue siendo, para la Unión Europea, el espacio natural donde deben de resolverse las cuestiones de integración social.

En segundo lugar, todo indica que el papel que se concede a las instituciones públicas con respecto a la empleabilidad, consiste en fomentar la mejora de las condiciones individuales de las personas para que puedan acceder al mercado de trabajo en igualdad de oportunidades. Puesto que se reconoce que existen discriminaciones en relación con el acceso al trabajo que afectan fundamentalmente a una serie de colectivos como las mujeres, los jóvenes, los mayores y los discapacitados, el esfuerzo de la acción social de inserción se plantea dirigido a mejorar las competencias, la empleabilidad de estos colec-

4. En este sentido, es necesario traer a colación el libro de C. Offe (1990), Contradicciones del Estado de Bienestar, Madrid, Alianza. En ese texto, el autor nos explica bien que la insistencia en el recurso trabajo como forma de inclusión social axiomática no hace más que recoger una vieja critica de la derecha económica y política, que considera que las «transferencias sociales» serían una desincentivación para el mercado de trabajo, lo que los anglosajones llaman las "poors traps", en el pensamiento de destacados autores como C. Murray, por poner un ejemplo.

5. Recordemos que, como decíamos, la propia Estrategia Europea para el Empleo, que se va construyendo a partir del libro blanco de Delors, tiene su sentido desde el momento en que la esfera política reconoce la incapacidad del mercado de trabajo para ofrecer empleo a toda la población demandante, a partir de los propios mecanismos de equilibrio de la oferta y la demanda predicados por el pensamiento económico neoliberal. 
tivos y personas para que accedan al empleo. Pero a eso queda confinado el grueso del papel que se reserva el Estado, a las administraciones públicas. Es decir, resulta llamativo el hecho de que, si la sociedad reconoce que es el trabajo asalariado la forma casi exclusiva de inclusión social, y, por otra parte, se constata la existencia de altas tasas de paro en el mercado de trabajo, el estado no proponga acciones de creación de empleo provenientes de sus propias áreas de influencia laboral. En este sentido, y citando varios estudios comparativos de evaluación de las políticas activas de empleo en diferentes países europeos, se apunta la creación directa de puestos de trabajo como una medida que ha ido cayendo en desuso por el escaso interés en la animación de los mercados laborales (según el enfoque de estos trabajos), dentro del conjunto de las políticas activas, y que si bien ha sido retomada en los últimos años por numerosos países europeos, no lo ha sido por su valor intrínseco, sino como mecanismo para controlar y reducir el número de prestaciones pasivas vinculadas a estos proyectos de creación de empleo público (Consejo Económico y Social, 2005: 65).

Volviendo a nuestro análisis, la empleabilidad se centra en la continua mejora y revisión del sistema de enseñanza, para adaptarlo a las nuevas condiciones de una sociedad del conocimiento (lo que implica la proposición de que haya participación de los capitales tanto públicos como privados en la enseñanza, como se viene señalando en algunos consejos europeos), y la propuesta de medidas de formación profesional ocupacional para los parados, en combinación con medidas de orientación laboral y profesional, así como la necesidad de realizar formación continuada en el empleo para prevenir el paro.

La empleabilidad, por lo tanto, queda definida a través de los textos de las diferentes cumbres mencionadas, y, tal y como la entendemos aquí, como un conjunto de adquisiciones tanto de tipo formativo y curricular, como de enfoque de las trayectorias laborales (saber tomar decisiones, saber orientarse en el mercado), que los individuos deben de ir forjándose para integrarse con éxito en el modelo laboral y social vigente. Debido al dinamismo de este modelo, a la vorágine con la que actúan los mercados laborales y profesionales haciendo obsoletos conocimientos, cualificaciones y profesiones en el seno de los cambios que nos llevan a una nueva sociedad de la información y del conocimiento $^{6}$, la sociedad asume la necesidad de que sus instituciones intervengan apoyando la mejora de la empleabilidad de sus ciudadanos, a través de formación, de servicios de orientación y otras medidas, pero haciendo recaer, en última instancia, la responsabilidad de la ubicación social y laboral de manera casi exclusiva en la esfera individual, ignorando las variables estructurales de los mercados de trabajo y de las políticas sociales que también intervienen en estos procesos, como explica muy bien Serge Ebersold (2004).

6. Quiero resaltar que este orden de cosas forma parte del discurso de la economía y del propio discurso europeo que situamos en la base de este trabajo, donde podrían estar hiperbolizados algunos elementos de los descritos. En cualquier caso, mi investigación no puede postularse sobre la adecuación de esta forma de ver la evolución laboral actual. 
En tercer lugar, vemos como, desde el Consejo de Amsterdam de 1997, se vienen planteando las políticas activas de empleo (solución encontrada en términos de intervención social, que se deriva del concepto de empleabilidad) como el tipo de políticas adecuadas para conseguir los objetivos europeos en términos de empleo y de cohesión social, sin, al mismo tiempo, romper con las disciplinas presupuestarias de la Unión Europea. De hecho, podemos constatar la consolidación de estas políticas activas de empleo, si estudiamos uno de los documentos recientes donde se hacen recomendaciones específicas sobre la política de empleo a seguir por la Unión Europea y que reafirma la tendencia expuesta. De la recomendación del Consejo sobre políticas de empleo en el año 2004, podemos hacer el siguiente extracto, que creo que se explica por sí mismo:

- Mejorar la capacidad de adaptación de los trabajadores y de las empresas, promoviendo al mismo tiempo la seguridad y la flexibilidad en el mercado laboral, creando más empleo y de mejor calidad y mejorando la productividad.

- Incitar más a las personas para que se incorporen y permanezcan en el mercado laboral y lograr que el trabajo sea una verdadera opción para todos, definiendo unas estrategias globales de envejecimiento activo (lucha contra la jubilación anticipada, organización más flexible del trabajo, valorización de las capacidades y oferta de formación), garantizando servicios personalizados a todos los solicitantes de empleo y haciendo que el trabajo sea más rentable.

- Invertir más, y de forma más eficaz, en capital humano y aprendizaje permanente, distribuyendo mejor los costes entre los poderes públicos, las empresas y las personas, desarrollando la oferta de formación, sobre todo para los trabajadores poco cualificados o mayores, reduciendo el abandono escolar y haciendo que la enseñanza superior se ajuste mejor al mercado laboral.

Puede verse perfectamente como se produce el binomio entre flexibilidad de los mercados de trabajo ${ }^{7}$, considerada como prerrequisito para el crecimiento económico, y necesidad de desarrollo de la formación y de las medidas de política activa de inserción laboral, cuestión que puede apreciarse en la frase final del segundo párrafo citado. No cabe duda de que la expresión: «haciendo que el trabajo sea más rentable», referida a la propia oferta laboral,

7. Entendemos aquí por flexibilidad laboral al conjunto de procesos legales (reformas laborales, por ejemplo) y de evoluciones en el marco de las relaciones laborales de las últimas décadas que han significado la necesidad de que la oferta de trabajo se adaptara y se adapte a los imperativos de la demanda, en pos de una archirrepetida necesidad de competitividad en un escenario económico marcado por el dinamismo de la globalización. Un excelente ejemplo a consultar para informarse sobre el significado de la flexibilidad en España en el marco de las reformas laborales lo encontramos en F. Valdés Dal-Ré (1999), «La flexibilidad del mercado de trabajo», El trabajo del futuro, Madrid, Editorial Complutense. 
recoge el sentir de los enfoques de política económica que consideran que las transferencias sociales y, por lo tanto, las llamadas «políticas pasivas de empleo» (como, por ejemplo, las tasas de cobertura de las prestaciones por desempleo) ejercen una influencia negativa perniciosa en la búsqueda de trabajo de los parados (Layard et al., 1994), tal y como ya hemos señalado con otros autores.

\section{La visión europea de los estados de bienestar activos}

¿Con qué argumentos y de qué forma se están enfocando las políticas sociales en relación con el Estado de bienestar? Esto nos servirá, tal y como planteamos, para intentar concluir si es hacia una Europa más centrada en el papel del estado como mediador de las crisis de lo social o, de otra forma, si es hacia una Europa más de mercado (predominio del empleo asalariado como forma prioritaria exigida a todos, integrados, vulnerables y excluidos, para un exitoso proceso de inserción social), en torno a la cual se están construyendo los parámetros de los modelos actuales de política y cohesión social. Vemos que los intentos de constituir una Europa social, de dotar a la construcción europea de una dimensión social y ciudadana que vaya más allá de la mera comunidad de interés económico, se encuentran ya en germen desde el Tratado Constitutivo de la Unión, que, en su artículo 136, declara como principales objetivos de la Unión Europea en política social los siguientes:

La Comunidad [la Unión Europea] y los Estados miembros [...] tendrán como objetivo el fomento del empleo, la mejora de las condiciones de vida y de trabajo, a fin de conseguir su equiparación por la vía del progreso, una protección social adecuada, el diálogo social, el desarrollo de los recursos humanos para conseguir un nivel de empleo elevado y duradero y la lucha contra las exclusiones.

Ya en esta declaración de objetivos podemos intuir algunas de las características de la definición de la política social europea, que, con su desarrollo posterior, se van manifestando fundamentales, como es la gran vinculación entre las cuestiones de discriminación en el mercado de trabajo y el fenómeno de la exclusión social, como también la estrategia prioritaria de fomento de los recursos humanos (¿qué son, si no, las políticas activas de empleo?) para enfrentarse al fenómeno de la exclusión social.

Algunos de los pasos que se han ido dando en la línea de la constitución de lo que hoy ya se llama "la Europa social», son los que abordamos a continuación. Entre los años 1995 y 1997, se desarrolló un programa de acción social a medio plazo, que arrancaba de la voluntad de la UE ya expresada en los libros blanco y verde de política social en 1993, de modernizar la política social europea y crear una política social «avanzada», tales eran los términos empleados. De modo que podemos datar en estos primeros años noventa las primeras referencias que se hacen a la necesidad de «modernizar» las políticas sociales, tal y como también se refleja en Consejo Económico y Social (2001). 
Las políticas sociales modernizadas que se proponen, se inscriben en la necesidad de buscar el equilibrio entre los objetivos de competitividad económica en un mundo globalizado y el mantenimiento de la cohesión social y los niveles de bienestar que caracterizan a Europa y, por otra parte, nos distinguen de los Estados Unidos. De hecho, en la mayoría de los objetivos, suelen plantearse de manera conjunta dos partes que, desde algunas teorías, podrían verse como contradictorias: el fomento de la competitividad y la cohesión social.

Así es exactamente como está planteado en el Consejo de Lisboa (2000) el objetivo general que se fija la UE, que habla explícitamente de hacer de Europa la «[...] economía basada en el conocimiento más competitiva y dinámica del mundo, capaz de sostener el crecimiento económico con más y mejores puestos de trabajo y mayor cohesión social». En esta cumbre, se habla explícitamente de cual es el objetivo de la política de inclusión, que es definido como «abordar el problema de la exclusión social en sus orígenes y contribuir a que la gente se autoayude». Se hizo hincapié en la modernización del modelo social europeo y en el desarrollo del Estado de bienestar activo. Se reafirmó en Lisboa el compromiso de seguir avanzando en el proceso de reforma económica (mejora del crecimiento económico, aumento de la flexibilidad de los mercados laborales, aumento de la competitividad), el empleo y la cohesión social, y se ratificó el compromiso respecto a una nueva agenda económica y social para el año 2000 y siguientes, desde las claves de avanzar en la economía basada en el conocimiento y en la creación de una Europa electrónica, haciendo referencia a la necesidad de que Europa y su ciudadanía no pierdan el tren de las nuevas tecnologías de la información en las que se pretendería que se basaran los pilares de la creación de riqueza del modo de producción postfordista y globalizado en el que estamos inmersos.

Será en la Cumbre de Niza de diciembre del 2000 donde se enraíce plenamente el nuevo contenido social de la Estrategia Europea de Empleo, que también se verá reforzado en Estocolmo al año siguiente, dentro del marco general de continuismo en el que se mueve el avance comunitario en estos ámbitos (Barbier, 2004: 8).

Posteriormente, en comunicación de la Comisión al Consejo Europeo, se desarrolla la Agenda de Política Social (2005-2010) ${ }^{8}$. Esta agenda, en comunión con la tendencia al desarrollo de lo que venimos aquí planteando en cuanto a hacer depender los objetivos sociales de la política económica, planteaba sus objetivos sobre dos ejes centrales: el pleno empleo, en el marco del objetivo de la prosperidad, y la igualdad de oportunidades y la inclusión social, en el marco del objetivo de la solidaridad.

Resumiendo lo tratado en relación con la exclusión y el Estado de bienestar, parece que está muy justificado considerar que el discurso europeo se cen-

8. Comunicación de la Comisión (2005), Agenda de Política Social [COM 33 finalno publicada en el diario oficial], obtenida del sitio web Scadplus, sobre documentación europea. 
tra en conectar siempre la lucha contra la exclusión social, vinculando ésta (como ya hemos demostrado en otros lugares) al modelo económico vigente y a sus resultados. El concepto de exclusión social enlaza, y aparece en el discurso, de la mano de otro concepto que gozaba de una vigencia anterior: el concepto de discriminación laboral. Por lo tanto, todo indica que la inserción por lo económico y el empleo serían la piedra angular del actual modelo de política social europea. Siendo esto así, la Estrategia Europea de Empleo constituiria el eje central sobre el que giraría la política social europea, y las medidas sociales que se diseñarían y se propondrían para la integración sociolaboral de los individuos. Luego hay que decir que de los ámbitos fundamentales de intermediación entre la situación de exclusión y la de integración de los individuos de los que partíamos en este análisis: el mercado y el Estado, la Unión Europea opta claramente por primar y revitalizar el primero frente al segundo.

\subsection{Efectos del lenguaje común socializador: el Estado de bienestar activo y la dimensión de lo local}

Nuestra hipótesis en este trabajo es que este lenguaje común con efectos socializadores, como lo llama Barbier (2004), que es la Estrategia Europea de Empleo, está planteando la necesidad de remodelar y reformar las instituciones del mercado y del Estado de bienestar, en función de la urgencia de encarar una cuádruple crisis que puede leerse en el discurso de la Unión Europea: una crisis de la economía (apelación a la necesidad continua de aumentar las tasas de crecimiento, siempre insuficientes para acometer políticas sociales de redistribución de la renta), una crisis del mercado de trabajo y del paro (se demanda continuamente a la necesidad de flexibilizar los mercados de trabajo, en pos de la competitividad), una crisis demográfica y una crisis del Estado de bienestar (entendido en el sentido keynesiano del término), crisis que, evidentemente, se encuentran vinculadas y postuladas entre sí.

Los nuevos perfiles de la política social y de empleo que se proponen pueden entenderse como el tránsito de los modelos históricos de bienestar, hacia lo que podríamos denominar como estados de bienestar activos, y que apelan, según la hipótesis que aquí se defiende, a la activación de varias instancias:

- A la activación de los individuos, fundamentalmente de los parados, que han de actualizar sus cualificaciones y recurrir a las políticas activas de empleo que se despliegan para ellos, a fin de conseguir su integración en el mercado laboral y, por ende, en la sociedad. La activación del individuo va aparejada a fomentar su participación, a crear personas activas, dinámicas, participativas, que sean las principales protagonistas de su proceso de inclusión, en el que serán acompañadas por el estado a través, fundamentalmente, de mecanismos de apoyo al empleo, como son las políticas activas de empleo. Aquí es central en el discurso socializador de la Estrategia Europea de Empleo el concepto de «empleabilidad» que hemos señalado anteriormente con más detalle. 
- A la activación de la sociedad civil, para que la lucha contra la exclusión social ante la que el Estado de bienestar keynesiano se habría mostrado insuficiente o incapaz de resolver, parta de la propia solidaridad ciudadana, representada fundamentalmente por el papel de las organizaciones no gubernamentales y sin ánimo de lucro, que suponen el gran motor de la sociedad para enfrentarse a la exclusión. El término partenariado ${ }^{9}$ designa a la vez un proceso, el de la necesidad de poner en marcha nuevas metodologías de trabajo en lo social, donde los proyectos de intervención se aborden de forma integral y por parte de todos los agentes implicados en un territorio. Por otra parte, también hace referencia al conjunto de actores reconocidos como solventes para intervenir en la cuestión social. En este caso, se hace un llamamiento sobre todo a las entidades no gubernamentales, por su pretendida mayor proximidad a los problemas de las personas en situaciones de vulnerabilidad y exclusión. Por otra parte, los agentes sociales, tanto sindicatos como representantes patronales, también constituyen actores clave en el desarrollo de estas políticas, con un rol coordinador por parte de las instituciones políticas y administrativas.

- A la activación de lo local, como espacio preferente de la lucha contra el desempleo y la exclusión social. Por lo tanto, hemos de entender, en mi opinión, que los estados nacionales asumen implícitamente su incapacidad para combatir los problemas de la cuestión social desde el ámbito centralizado y universalista de la función de gobierno y, por lo tanto, presuponen que es en el ámbito de lo local, de la cercanía a los problemas concretos de los ciudadanos, del desempleo local específico, donde se tienen que activar las energías de la comunidad, para encontrar fórmulas imaginativas e innovadoras de integración social.

Lo local es el espacio donde ha de materializarse, de manera fundamental, otro concepto que está muy asociado al nuevo modelo social europeo: el de partenariado, al que ya nos hemos referido. Los nuevos planes de inclusión, que se concretan primero a nivel nacional, luego regional y, finalmente, local, recogen la necesidad que, frente a las acciones descoordinadas que hasta ahora han regido entre las entidades dedicadas al trabajo de lo social, existan planes o proyectos coordinados para el tratamiento conjunto de un área, como las áreas necesitadas de transformación social. La idea, por lo tanto, sería: la activación del tejido social para que, al final, ésta llegue a crear la propia activación y participación del individuo.

Si bien no hay que subestimar el potencial de creación de nuevas redes de sociabilidad y de nuevas formas de subjetividad y de inclusión social que puede desarrollarse a partir de la entrada en escena de este nuevo modelo de bienestar, que abre la acción social a lo local, al tejido asociativo y a la combinación de acción pública y privada, también tenemos que señalar los riesgos que algu-

9. Es un concepto clave para el desarrollo de la «Europa Social» y que está siendo fuertemente promovido y difundido por la Comisión Europea. 
nos autores postulan como contrapartida. El principal riesgo que se apunta es el de la pérdida del sentido universal de las políticas sociales, señalado, por ejemplo, por Alonso (1999). El recurso a lo local, a la solidaridad civil y a la activación individual podría contener un elemento pernicioso: el de la desresponsabilización del estado en las cuestiones relativas a la exclusión social, en definitiva, el riesgo de que el estado y, por tanto, los derechos ciudadanos pudieran ensayar una cierta estrategia de retirada en el sentido expresado. Esto podría afectar a los derechos de ciudadanía y a la capacidad del nuevo modelo económico para mantener la cohesión social (Alonso, 1999).

El hecho de centrar las estrategias de empleo y de abordaje de la exclusión social en lo local, podría llevar a generar fuertes desigualdades entre los ciudadanos, en función de que vivan en un lugar o en otro de los territorios nacionales. Esto, sin duda, ya está muy consolidado en la actual Unión Europea, donde el informe de Paugam y Gallie (2001), elaborado a petición de la propia Comisión Europea, ya señala esta problemática con absoluta rotundidad. Los niveles de protección social y de bienestar son mucho más elevados en los países del Norte, cuyo paradigma sería Suecia, que en los países del Sur, bien representados por Portugal ${ }^{10}$. Además, no debemos de olvidar que, con posterioridad a ese informe, se ha producido el ingreso en la Unión de doce nuevos socios, muchos de ellos países como Hungría o Rumanía, que están en condiciones de renta y de riqueza muy desiguales frente a la media europea. Todo esto permitiría formular, entendemos, la siguiente hipótesis: el intento de generalización de los principios de un tipo de política social y de empleo que pueden ser eficaces en unos contextos determinados puede conllevar el aumento de las desigualdades y la ineficacia e ineficiencia de estas políticas en otros ámbitos nacionales o regionales de su marco de aplicación, e incluso conllevar la existencia de incoherencia y efectos perversos o funciones latentes en principio no perseguidas por sus actores.

\section{Algunos balances sobre la Estrategia Europea de Empleo}

Aunque no vamos a caer en el enfoque simplificador que pretende que la Estrategia Europea de Empleo es una concepción de política laboral directamente emanada de la influencia de las políticas de empleo estadounidenses del workfare $^{11}$ y una resultante exclusiva del enfoque neoliberal en materia eco-

10. Distintos estudios comparativos como los efectuados por Alujas Ruiz o Luis Fina Sanglas, sobre el que abundaremos más abajo, también llegan a similares conclusiones en cuanto a la desigualdad de concreciones y resultados de las políticas de empleo, en este caso, entre los distintos estados nacionales sometidos a la influencia de un mismo enfoque de políticas de empleo.

11. Jean Claude Barbier (2001: 6) define el concepto de workfare de la siguiente forma: "Desde entonces los programas del workfare siempre han pivotado sobre una característica constante: la imposición de una obligación o contrapartida laboral a los individuos solicitantes de medidas de ayuda social» (la traducción al castellano es del autor del presente artículo). 
nómica de trabajo, tal y como nos advierte Barbier (2001), sí que podemos constatar de la lectura de los textos europeos al respecto, la importante influencia de las argumentaciones económicas dominantes en el contexto de definición de estas políticas de la Estrategia Europea de Empleo. Argumentaciones económicas que parecen plantear la necesidad de reorientar determinadas concepciones sobre la exclusión social y sobre el papel que el Estado tiene que desempeñar en el proceso de la integración social.

Diversos informes, entre ellos el ya citado de Paugam y Gallie (2002), están demostrando que, en tiempos de crecimiento de la riqueza económica, se va instalando entre la población una visión sobre la exclusión social que tiende a encontrar las causas de ésta más en las características de los individuos que en las condiciones de desigualdad e injusticia social que son estructurales en una economía de mercado. También en esa misma línea, y como una de las conclusiones generales, el informe del 2002 del Observatoire Nationale de la Pauvreté et de l'Exclusion Sociale francés, señala que las personas se muestran menos comprensivas con las posibles causas sociales de la pobreza y de la exclusión social, cuanto que pertenencen a las capas salariales más altas y están menos en contacto, directo o indirecto, con este tipo de situaciones sociales. No se ven razones para pensar que estudios de corte general lanzados en España (recordemos que no existe en nuestro país un observatorio de naturaleza semejante hasta el momento) pudieran arrojar resultados muy diferentes en cuanto a representaciones sociales sobre la exclusión, ya que cabe pensar que lo que anima a esta visión colectiva de la exclusión, si puede hablarse propiamente de ella, es la existencia de un modelo social cada vez más individualizado en el que España estaría participando de la misma forma que el país vecino.

Si nos remitimos al informe sintético de Anna Diamantoupoulou (2002: 133-134), encontramos una serie de conclusiones de gran interés que refuerzan los argumentos que hemos ido desgranando a lo largo del análisis que hacemos de la Estrategia Europea de Empleo. Se recomienda que las políticas sociales y de empleo se centren lo máximo posible en el ciudadano, que sean lo más cercanas posible a su ámbito de decisión y que se establezcan mecanismos ágiles y diáfanos para la distribución de las responsabilidades entre los distintos partenariados sociales. Entendemos que esto va en la línea de un reconocimiento explícito de los problemas de coordinación reales que han aparecido a la hora de implementar el método abierto de coordinación (MAC) y la filosofía del partenariado.

Y, como colofón de lo anterior, traeremos a colación las conclusiones principales a las que llega el informe conjunto sobre empleo (2005), editado también por la Comisión, que no hace más que mostrar una línea continuista con los textos citados anteriormente y, por tanto, ratificar las líneas de fuerza que defiendo en este trabajo. En principio, el informe, que analiza los logros y la tarea que queda por hacer para desarrollar la Estrategia Europea de Empleo, sigue sosteniendo el argumento general de lo decisivas que han resultado las reformas en la línea de flexibilización de los mercados laborales europeos para lograr la resistencia que ha mostrado el empleo y las tasas de paro ante la crisis econó- 
mica que ha sufrido Europa entre los años 2001-2004, tal y como se pronuncia $^{12}$. Se insiste, además, en que sólo se podrá crear más empleo y aumentar el crecimiento económico por la vía de seguir acometiendo reformas del mismo calibre, pero en sectores donde todavía se ha avanzado muy poco en este sentido, como son el caso del mercado de servicios y el mercado financiero.

El discurso se traza a base de conceptualizaciones vagas, que permiten, por tanto, una independencia relativa en su interpretación. Ahora bien, esto dentro de un marco de política macroeconómica, donde las recomendaciones sí que son mucho más concretas y adoptan un tono más prescriptivo. En cuanto al planteamiento conjunto de objetivos que pueden considerarse contradictorios, este texto también resulta ejemplificador. Así, en el capítulo en que abordan los esfuerzos realizados por los estados miembros para mejorar la capacidad de adaptación de los trabajadores y de las empresas, se hace mención expresa al esfuerzo cada vez mayor desplegado por estos estados para conciliar el equilibrio entre la seguridad y la flexibilidad. Por supuesto, dentro de la tendencia al empleo de declaraciones vagas y más centradas en los significantes que en los significados, no se dice de qué forma los estados miembros consiguen poner en marcha esta tarea y sus supuestos logros.

En cuanto al enfoque que concibe las políticas activas como sustitutivas de las pasivas, también hay numerosas recomendaciones que llaman al rigor de los estados miembros, en el sentido de que hagan más esfuerzos para facilitar que el trabajo resulte más atractivo a los que están sujetos a medidas de apoyo económico, como subvenciones por desempleo, rentas mínimas, etc., e incluso se deplora que no se hagan suficientes esfuerzos en esta materia. Por lo tanto, si bien puede decirse que las políticas sociales y de empleo europeas difieren de las estadounidenses y mantienen unas señas de identidad que se expresarían, por ejemplo, en la filosofía expresada en la Agenda Social Europea, no es menos cierto que la tendencia de estas políticas sociales y de empleo en Europa ha girado, como hemos mostrado, en el sentido de la influencia del workfare americano, tal y como lo hemos definido con antelación ${ }^{13}$.

\section{Conclusiones}

En definitiva, todo indica que la formulación de la política de empleo en la Unión Europea actual, basada esencialmente en las políticas activas de empleo

12. En este mismo informe, se aporta bibliografía para aquellos que desearan conocer la amplitud estadística de los datos económicos y de empleo en el periodo de los últimos años; información que puede descargarse de Internet en la siguiente dirección: http:/Europa.eu.int/comm/empoyment_social/analysis/employment_fr.htm

13. Podemos señalar también a Andersen y Hesen (2002) como autores que, desde el campo de la teoría académica, dan prueba en numerosos lugares de la relación entre política social y trabajo en el marco de la constitución de los derechos efectivos de ciudadanía. 
como opción prioritaria e incluso sustitutiva de las políticas pasivas, corre normalmente de manera paralela a las exigencias en materia de disciplina económica, flexibilidad de los mercados laborales, facilitación de la movilidad de los capitales, etc., aunque ambas esferas puedan tener, y todo indica que las tienen, una independencia relativa entre sí, en el seno de la formulación de políticas comunitarias.

Creemos que el discurso que emana de la ingente cantidad de textos, directivas, directrices europeas en materia de empleo y cohesión social, no estaría exento de ambivalencias. Se diría que, en cada peldaño, en cada receso para pasar a una fase posterior, se encuentra toda una jerga optimista y legitimadora de los resultados obtenidos en materia económica y de empleo, que justifica el avanzar, el apretar el acelerador, en una dirección y en rumbos que parecieran estar marcados ya, de manera mimética, de antemano. La Estrategia de Lisboa se construye sobre los avances y siguiendo y profundizando en los impulsos emprendidos ya en Amsterdam y Luxemburgo. Se profundiza en un tipo de política de empleo, en este caso, la política activa de empleo, porque se considera que da buenos resultados. Pero, al mismo tiempo, y paradójicamente, se reconocen los fuertes límites que ha encontrado la Estrategia Europea de Empleo para producir resultados positivos (reducción adecuada de las tasas de paro). Cada impulso adelante parece implicar, también, un reconocimiento al hecho de que el modelo económico y sus triunfos no se están trasladando de manera suficiente al marco de la creación de empleo y la cohesión. Pero, paradójicamente, en lugar de proponer medidas alternativas, se apuesta por seguir en la misma ruta, pero aumentando la velocidad.

Por lo tanto, si al principio nos formulábamos la pregunta con respecto a la naturaleza de este lenguaje común socializador en materia de empleo que supone la Estrategia Europea, tenemos que decir que todo apunta a que el acento se está poniendo, desde Europa, en el mercado de trabajo por encima del carácter protector del Estado de bienestar. La influencia de las políticas de empleo del workfare, sin caer en determinismos ideológicos que aportarían explicaciones maniqueas sobre el triunfo unilateral e irreversible de una visión neoliberal de las políticas sociales y de empleo, sí parece constatarse con bastante claridad. Tal es así, que hemos podido comprobar cómo se van conectando los discursos y las estrategias en materia económica con la cohesión social y las políticas activas de empleo. Todo ello podría estar reconfigurando la propia visión que tendrian las elites directivas sobre el Estado de bienestar, y parece que pudiéramos estar en un periodo de tránsito donde se muestran signos de otra forma de hacer y de entender la política social, que tendería a poner el acento en el concepto de empleabilidad, frente al modelo clásico del Estado de bienestar keynesiano.

Algunas de las características de estos nuevos contextos de las políticas sociales serían el axioma de hacer pasar la inserción social por la inserción a través de lo económico, de manera que las políticas activas de empleo ocuparían un papel central. En torno al concepto de empleabilidad que parece emerger con fuerza en este periodo, se recomponen los actores y las responsabilidades de unos y otros en relación con la cuestión social. La exclusión del mercado 
de trabajo, de la sociedad, corre el riesgo de desconectarse de explicaciones de tipo estructural y pasa a ser, esencialmente, responsabilidad de los sujetos mismos. El riesgo de todo ello es que la sociedad pueda estar pidiendo a los sujetos lo que éstos no puedan darle, y se acabe culpando a los carecientes de su propia situación de carencia. Pero la sociedad y el estado no dimiten por completo. El estado y otra serie de actores sociales asumen aquí un rol en el intento de mantener la cohesión social. El estado se convierte, fundamentalmente, en un coordinador de esfuerzos institucionales (ONGs, agencias locales de empleo, etc.), que, en lo sucesivo, son teorizados como mucho más efectivos y equitativos si se ofrecen desde un marco local. Sin duda, esto abre interesantes horizontes a la experimentalidad en materia de inserción laboral y social, a la creación de sinergias, pero, al mismo tiempo, no podemos dejar de señalar, como lo hacen numerosos autores, el peligro de individualización y de segmentación de las sociedades y de los mercados laborales que estos enfoques podrían suponer.

\section{Referencias bibliográficas}

Alonso, L.E. (1999). Trabajo y ciudadanía. Madrid: Trotta.

Andersen, G. (2002). Changing Labour Markets, Welfare Policies and Citizenship. Bristol: Policy Press.

BARBIER, J.C. (2001). Welfare to work policie in Europe: the current challenges of activation policies. París: Centre d'Études de l'Emploi, Document de Travail, 11, 124.

- (2004). La estrategie europeénne pour l'emploi, génese, coordination communautaire et diversité nacional. París: Raport de Recherche pour la DARE (Ministére de Travail).

BECK, U. (1997). ¿Qué es la globalización: falacias del globalismo, respuestas a la globalización. Barcelona: Paidós.

CASTEL, R. (1997). La metamorfosis de la cuestión social: una crónica del salariado. Buenos Aires: Paidós.

Castillo, J.J. (1998). A la buisqueda del trabajo perdido. Madrid: Tecnos.

- (1999). El trabajo del futuro. Madrid: Complutense.

Commision des Communautés Européenes (2005). Project de rapport Conjoint Sur l'emploi. Bruselas.

Comisión Europeénne, Travail et Affaires Sociales (2006). Principales étapes de la SEE. Bruselas. [http://ec.europa.eu/employment_social/employment_strategy/develop_fr.htm]

Consejo EConÓmico y Social (2001). La Estrategia Europea de Empleo. Madrid.

- (2005). Desequilibrios ocupacionales y políticas activas de empleo. Madrid.

DeLORS, J. (1993). Croissance, compétitivité, emploi. Les dédits et les pistes pour entrer dans le 21eme Siécle. Bruselas: Comisión Europeénne.

Diamantoupoulou, A. (ed.) (2002). Informe sobre el estado de la estrategia europea para la inclusion social. Bruselas: Unión Europea.

EBERSOld, S. (2004). «L'insertion ou la délégitimation du chomeur». Actes de la Recherche en Sciences Sociales, 154, 94-102.

FINA, L. (2001). Mercado de Trabajo y Politicas de Empleo. Madrid: CES. 
Gough, I. (2000). Global capital, Human Needs and Social Policies. Nueva York: Palgrave.

JoHNSON, N. (1990). El Estado de bienestar en transicion: la teoría y la práctica del pluralismo de bienestar. Madrid: Ministerio de Trabajo y Seguridad Social.

KOK, W. (2004). Hacer frente al desafio: La Estrategia de Lisboa para el crecimiento y el empleo. Informe de la Comisión Europea. Bruselas. [http://ec.europa.eu/growthandjobs/pdf/kok_report_es.pdf]

LAYARD, R.; NICKELL, S. y JACKMAN, R. (1994). El paro: los resultados macroeconómicos y el mercado de trabajo. Madrid: Ministerio de Trabajo y Seguridad Social.

MAUGER, G. (2001). «Les politiques d'insertion. Une contribution paradoxale á la desestabilisation du marché du travail». Actes de la Recherche en Sciences Sociales. París, 137-149.

Ministerio de Trabajo y Seguridad Social (1995). La politica de empleo en España: Informe de base sobre instituciones, procedimientos y medidas de política de empleo. Madrid.

Observatoire Nationale de la Pauvreté et de l’Exclusion Sociale (2002). Rapport 2001-2002. París: La documentation française.

Offe, C. (1990). Contradicciones del Estado de Bienestar. Madrid: Alianza.

Paugam, S. y Gallie, D. (2002). Social Precarity and Social Integration. Bruselas: Rapport pour la Commission européenne base sur l'eurobaromètre, 56.1, junio.

RECIO, A. (1997). Trabajo, personas, mercados. Madrid: Icaria.

RIFKIN, J. (1999). El fin del trabajo: nuevas tecnologias contra puestos de trabajo: El nacimiento de una nueva era. Barcelona: Paidós.

Santos, A.; Montalbá, C. y Moldes, R. (2004). Paro, exclusión y políticas de empleo, aspectos sociológicos. Valencia: Tirant Lo Blanch.

SENNETT, R. (2000). La corrosión del carácter: Las consecuencias personales del trabajo en el nuevo capitalismo. Barcelona: Anagrama.

TAYlor-Gooby, P. (ed.) (2004). Making a European Welfare State? Convergences and Conflicts over European Social Policy. Oxford: Blackwell. 